\title{
A05
}

\section{Numerical Simulation of Capacitively Coupled Resistivity Imaging Measurements on Rock Samples}

\author{
S.S. Uhlemann* (ETH Zürich), O. Kuras (British Geological Survey) \& A.G. \\ Green (ETH Zürich)
}

\section{SUMMARY}

Estimation of electrical properties in permafrost studies can benefit from capacitive resistivity imaging (CRI), which overcomes the limitations of usual electrical resistivity imaging employing galvanic coupled electrodes. However, the response of these systems is not fully understood yet. We have used numerical finite element modelling to investigate the complex transfer impedance as measured by a quadrupole consisting of sensors with finite dimensions. Next to a parameter study over a homogeneous halfspace, also an actual experimental setup is modelled. The numerical results are compared to a quasi-static approximation based on point-poles and measured data, respectively. The parameter study shows that the real part of the complex transfer impedance can be approximated by the quasi-static approach in case the dipole separation is large compared to the sensor dimensions. It is shown that quantification of the imaginary part is difficult and depends strongly on the geometric setup. The results of the simulation of a $3 \mathrm{D}$ finite rock sample indicate that finite element modelling can be a practical tool for improving the understanding of the experimental data and for performing a better error analysis. 


\section{Near Surface \\ Geoscience 2012}

\section{Introduction}

Electrical resistivity imaging (ERI) is a widely used tool in near surface geophysics, which is now being applied to the investigation of permafrost processes at both field and laboratory scales (Krautblatter and Hauck 2007; Krautblatter et al. 2010; Kneisel et al. 2008). However, the performance of ERI in a permafrost environment is restricted by the galvanic coupling of the electrodes to the ground. This problem can be overcome by the use of a capacitively coupled system, which has particular benefits for sensor emplacement and the contact resistance of electrodes.

The application to archaeological studies of such an capacitively coupled approach and its equivalence to ERI are shown in Tabbagh et al. (1993); the underlying theory of this equivalence is reviewed in Kuras et al. (2006). However, both studies are based on a quasi-static approach employing point poles instead of sensors with finite dimensions.

Since no analytical solution exists for the latter case, we have employed numerical modelling and compared the results to the analytical solution for the quasi-static approach. The aim of the study is to understand the response of such a system, determine its limitations, and validate the use of conventional ERI tools for the interpretation of the acquired data.

We have used the AC/DC Module of Comsol Multiphysics ${ }^{\circledR}$ for the finite-element numerical modelling. Its implementation of quasi-static electrical and electromagnetic physics applies directly to our problem, such that parameter studies are possible.

\section{Analytical solution using a quasi-static approach}

The analytical solution using the quasi-static approach serves as a reference for the comparison with the numerical solution and for examining the error introduced by the discrepancy between point poles and finite size sensors. As shown in Grard (1990) and Kuras et al. (2006), the transfer impedance $Z$ can be derived using image sources and is given by

$$
Z=Z_{0}\left(1-K^{E S} \alpha\right)
$$

where $Z_{0}$ is the transfer impedance in free space, $\mathrm{K}^{\mathrm{ES}}$ is a geometric factor consisting of the pole distances and distances to the image source points, and $\alpha$ is a complex factor comprising the electrical properties of the subsurface. For an air-ground interface $\left(\varepsilon_{\text {air }}=1\right) \alpha$ is defined as

$$
\alpha=\rho \omega \varepsilon_{0}\left(\varepsilon_{r}-1\right)-i / \rho \omega \varepsilon_{0}\left(\varepsilon_{r}+1\right)-I
$$

and thus already shows the capability of the capacitively coupled quadrupole to image the resistivity distribution in the subsurface. The complex nature of this factor leads to a complex value for the transfer impedance, for which the real and imaginary parts will be discussed separately.

In Kuras et al. (2006), the relation between ERI and values obtained with capacitive resistivity imaging (CRI) is derived and it is shown that the real part of the complex transfer impedance is sufficient to estimate a dc apparent resistivity in case the signal is in phase.

\section{Model Setup and Parameter Study}

Using the AC/DC module in Comsol Multiphysics ${ }^{\circledR}$, we have simulated a capacitively coupled quadrupole over a homogeneous halfspace. By employing infinite elements at the boundaries of the domain, the typical assumption in geophysical halfspace problems of infinite extensions is satisfied (Butler and Sinha 2011). The quadrupole is modelled in a square equatorial configuration; hence dipole length and separation are equal. The model consists of the ground halfspace, while the actual sensors are located in a second overlying halfspace representing air. During the course of the parameter study, the sensor size is varied as well as the relative dipole length, sensor elevation and 


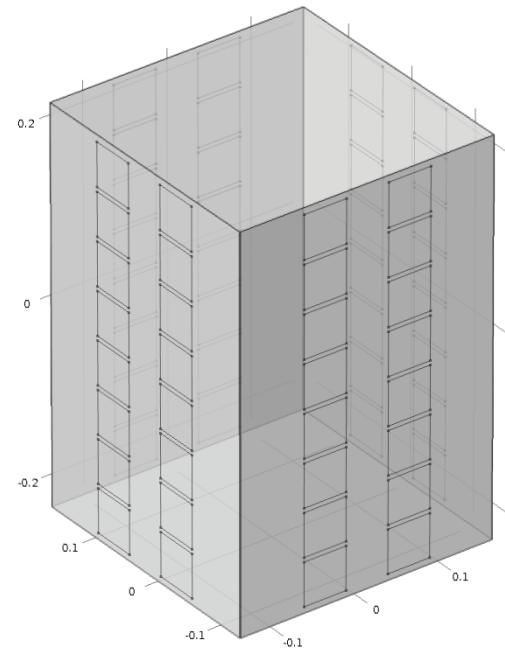

Figure 1 Model geometry for the simulation of the laboratory measurements in Comsol Multiphysics ${ }^{\circledR}$. halfspace resistivity; these are all factors that might vary in experimental setups or which cannot be determined exactly.

In a next step, actual laboratory experiments on a saturated rock sample of finite size (Kuras et al. 2011) are simulated. In Figure 1, the model setup is shown. The numerical results are compared with experimental data obtained with a recently developed prototype CRI system using 64 electrodes. A dataset of 688 different combinations of source and receiver dipoles was measured in the laboratory and is modelled here.

\section{Results}

Quadrupole model. Figures 2 and 3 show the results of comparing the numerical solution with the quasi-static analytical one. The difference between the analytical and numerical solutions is shown as a relative error.

The real part in Figure 2 clearly shows that with decreasing sensor size the error between a point pole assumption and a sensor with finite dimensions decreases, as one would expect.

Where this systematic geometric error is found to be of the same order or smaller than the anticipated measurement error, the use of the quasi-static approximation for further processing of the data is deemed reasonable.

The results for the imaginary part highlight the dependence of the solution on the chosen mesh. In contrast to the real part, the error between analytical and numerical solution is severe. The larger error for the results with constant mesh properties might be explained with a too coarse discretisation of the sensor domains for small widths, since with adapted mesh properties (blue lines), and hence better discretisation, the error is approximately halved. The strong dependence of the imaginary part of the transfer impedance on the quadrupole geometry is shown in Kuras et al. (2006); however this large
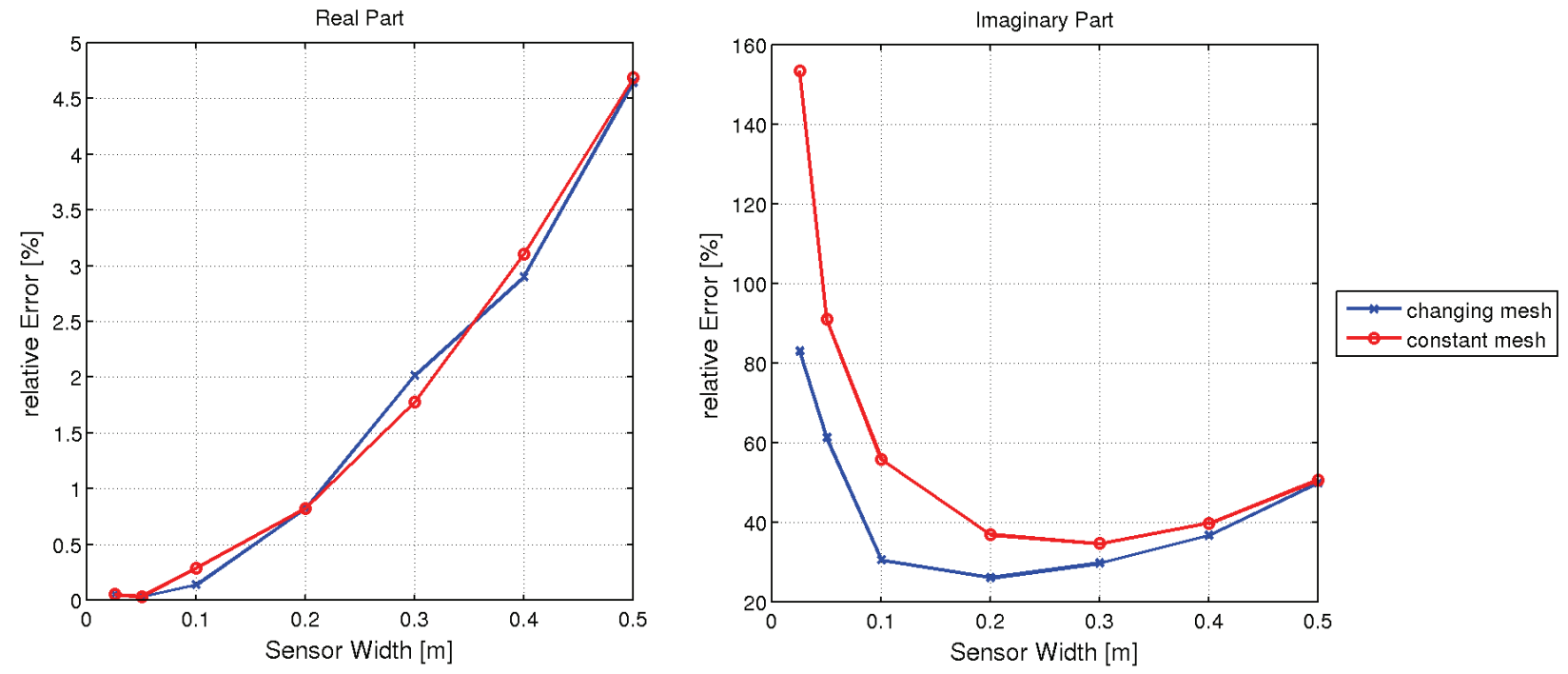

Figure 2 Comparison of real and imaginary parts of the numerical solution with the analytical one for different sensor widths. The difference is shown as relative error. The red line shows results calculated on meshes with equal properties, whereas the blue line shows results on meshes adapted to the sensor size. The dipole length is held constant at $1 \mathrm{~m}$ over a halfspace of $\rho=100 \Omega m$. 


\section{Near Surface \\ Geoscience 2012}
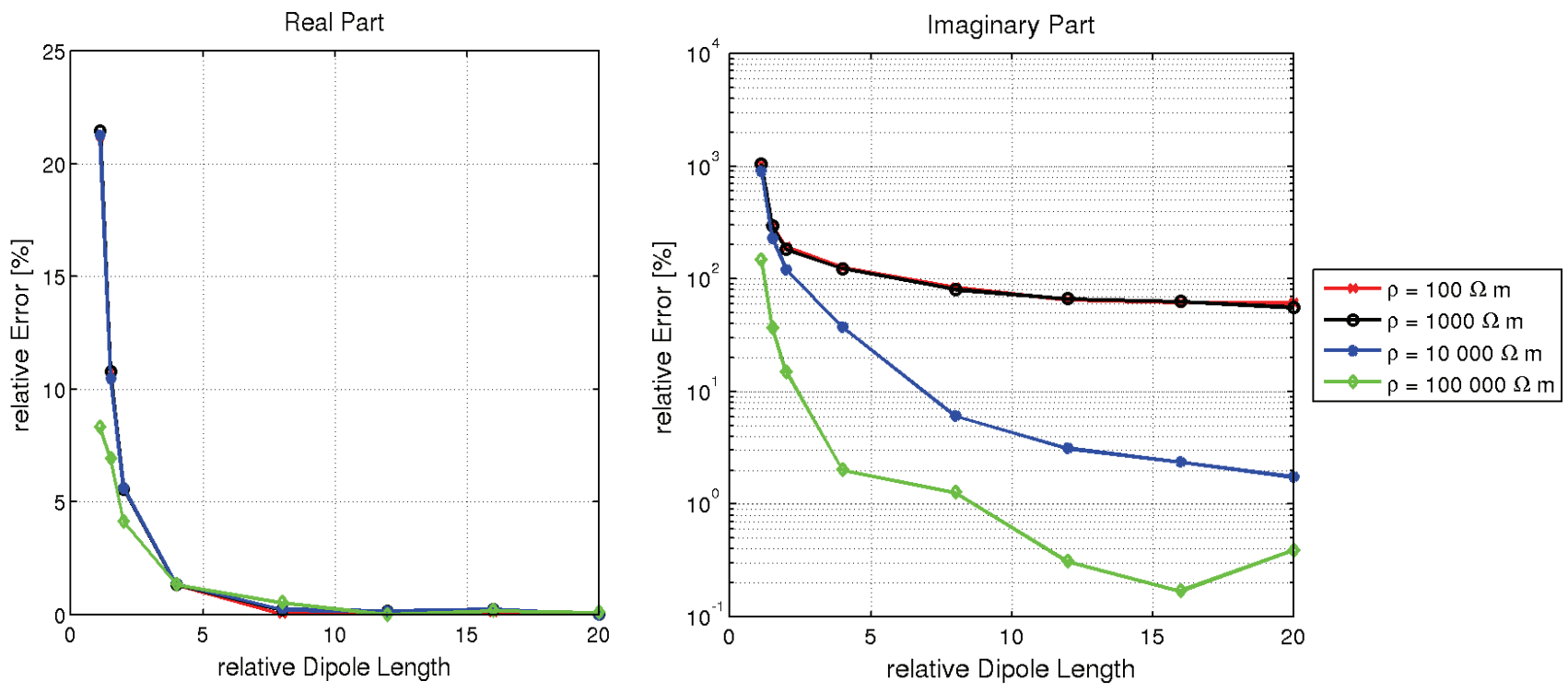

Figure 3 Comparison of real and imaginary parts of the numerical solution with the analytical one for different dipole lengths for a sensor width of $0.05 \mathrm{~m}$ and an operational frequency of 15 $\mathrm{kHz}$ for four different halfspace resistivities.

error between the numerical solution for sensors with finite dimensions and the analytical solution for point poles shows that the use of experimental data for the imaginary part of the transfer impedance may need to be limited to qualitative interpretation, especially when dealing with conductive material.

The dependence of the error on the halfspace resistivity and relative dipole length are shown in Figure 3. The real part is only minimally dependent on the halfspace resistivity, and small differences between the various resistivities are expected to be mesh effects. In contrast, the dependence on the relative dipole length is obvious; with decreasing dipole length the difference between the numerical and analytical solutions increases. Clearly, a point pole approximation is not valid for small dipole lengths. This fact has to be considered in experimental design, since the geometric error can be much larger than a typically expected measurement error of about $5 \%$.

In contrast to the real part, the imaginary part is found to depend on both resistivity and relative dipole length. The behaviour with respect to the dipole length is equal to the real part. With decreasing dipole length the error increases, since the approximation of point poles is violated. However, the dependence on the halfspace resistivity shows that with increasing resistivity the error reduces and can be of the same order as that of the real part for sufficiently large dipole separations. This indicates the possibility in resistive environments (e.g. permafrost) to use the imaginary part as additional information next to the real part. Nevertheless, care has to be taken when using these data since the imaginary part of the transfer impedance strongly depends on the geometric configuration of the sensors.

3D model of the rock sample. The resistivity of the sample in the numerical simulation of the laboratory experiment is defined by the inversion results of the experimental data. All experimental combinations of source and receiver dipoles were modelled and compared with the measured data to estimate whether the experimental outcome can be explained by the numerical modelling. In Figure 4, the measured potential is plotted against the modelled potential. Both the model and measurement errors are shown. The model error is orders of magnitude smaller than the measurement error (about $0.001 \%$ and $5 \%$, respectively), although the measurement error is so far only based on a repetition of the experiment, not on reciprocal measurements. Nor has a misplacement error of the electrodes been considered, which can be of the order of the measurement error (Oldenborger et al. 2005). Still, this comparison shows that the measured and modelled data follow the same trend, suggesting that 


\section{Near Surface Geoscience 2012}

numerical simulation is a viable tool for understanding the experimental data in greater detail. For example, an error analysis indicates that the electrodes at the boundaries show the largest difference between measured and modelled data.

\section{Conclusions}

Finite-element modelling of the response of a capacitively coupled quadrupole over a homogeneous halfspace revealed that the characterisation of the response by a quasistatic approximation is valid for the real part of the complex transfer impedance if the dipole length is large compared to the actual sensor dimensions. This relation does not depend on the halfspace resistivity. By comparison, the imaginary part was shown to depend strongly on the configuration setup and an approximation by the quasi-static

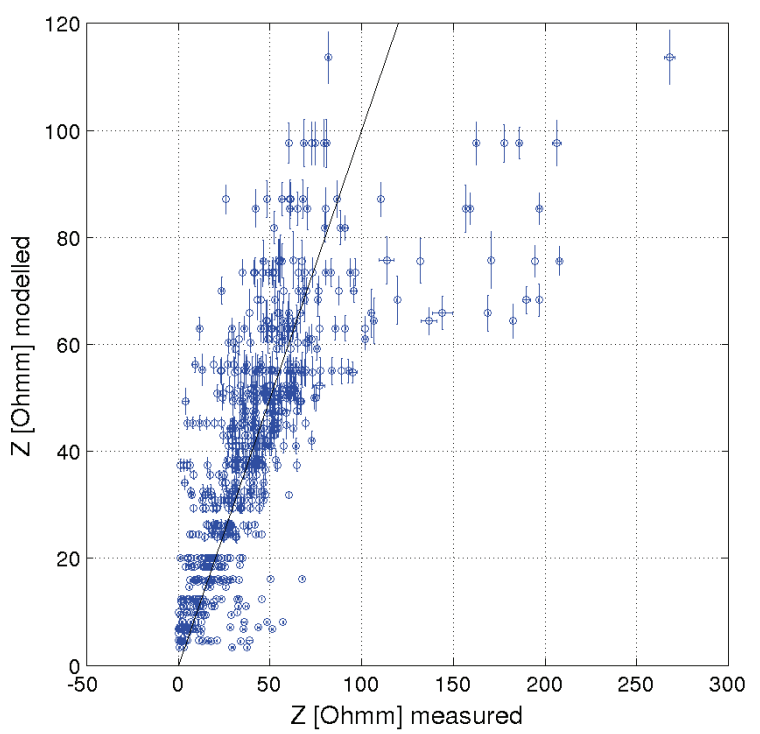

Figure 4 Comparison of simulated and measured data including error bars (error for simulated data exaggerated by a factor of 1000). approximation is only valid in the case of a large halfspace resistivity and large dipole separation. Modelling of a laboratory experiment highlighted the potential of numerical simulation for aiding and improving the understanding of the acquired data.

\section{References}

Butler, S.L. and Sinha, G. [2011] Forward modeling of applied geophysics methods using Comsol and comparison with analytical and laboratory analog models. Computers \& Geosciences, 1-9.

Grard, R. [1990] A quadrupolar array for measuring the complex permittivity of the ground: application to Earth prospection and planetary exploration. Measurement Science and Technology, 1, 295-301.

Kneisel, C., Hauck, C., Fortier, R. and Moorman, B. [2008] Advances in geophysical methods for permafrost investigations. Permafrost and Periglacial Processes, 19(2), 157-178.

Krautblatter, M., Verleysdonk, S., Flores-Orozco, A. and Kemna, A. [2010] Temperature-calibrated imaging of seasonal changes in permafrost rock walls by quantitative electrical resistivity tomography (Zugspitze, German/Austrian Alps). Journal of Geophysical Research, 115(F2), 115.

Krautblatter, M. and Hauck, C. [2007] Electrical resistivity tomography monitoring of permafrost in solid rock walls. Journal of Geophysical Research, 112(F2), 1-14.

Kuras, O. et al. [2011] Time-lapse Capacitive Resistivity Imaging - A Novel Methodology for the Monitoring of Permafrost Processes in Bedrock. Near Surface 2011 - the $17^{\text {th }}$ European Meeting of Environmental and Engineering Geophysics, Extended Abstracts, P35.

Kuras, O., Beamish, D. and Meldrum, P. [2006] Fundamentals of the capacitive resistivity technique. Geophysics, 71(3), G135-G152.

Oldenborger, G., Routh, P.S. and Knoll, M.D. [2005] Sensitivity of electrical resistivity tomography data to electrode position errors. Geophysical Journal International, 163(1), 1-9.

Tabbagh, A., Hesse, A. and Grard, A. [1993] Determination of Electrical Properties of the Ground at Shallow Depth with an Electrostatic Quadrupole: Field Trials on Archaeological Sites. Geophysical prospecting, 41(5), 579-597. 